\title{
$X X$. On some new auxiliary tables for determining the apparent places of the Greenwich stars
}

\author{
Francis Baily Esq. F.R.S. L.S. G.S. M.R.I.A.
}

To cite this article: Francis Baily Esq. F.R.S. L.S. G.S. M.R.I.A. (1827) XX. On some new auxiliary tables for determining the apparent places of the Greenwich stars, Philosophical Magazine Series 2, 1:2, 81-89, DOI: 10.1080/14786442708674232

To link to this article: http://dx.doi.org/10.1080/14786442708674232

Published online: 10 Jul 2009.

Submit your article to this journal $\square$

Џ Article views: 2

Q View related articles ๘ 


\title{
PHILOSOPHICAL MAGAZINE
}

\author{
$A N D$
}

\section{ANNALS OF PHILOSOPHY.}

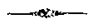 \\ [NEW SERIES.] \\ FEBRUARY 182 ?.
}

$\mathrm{XX}$. On some new auxiliary Tables for determining the apparent Places of the Greenvich Stars. By Francrs Bailx, Esq. F.R.S. L.S. and G.S. and M.R.I.A.

$$
\text { To Mr. Taylor. }
$$

1. N a late Number of your valuable Journal ${ }^{*}$, you have noticed the New Tables for facilitating the computation of Precession, Aberration and Nutation recently published by the Astronomical Society of London. These tables certainly present the most convenient mode of computing those quantities, when occasionally required: but they, by no means, supersede the utility and (in the present state of astronomy, I may say) the necessity of those Special Tables for the daily corrections of the Greenwich stars, which were computed also at the expense of the Astronomical Society, at the suggestion (I believe) of Mr. Herschel; and who has written an excellent Introduction to the same.

2. It is well known that Mr. Herschel's tables are intended only to be subsidiary to the formation of other tables of the corrections which a daily required in an active observatory: and to save the time and labour which must otherwise be employed in determining those quantities. And, in the computation of those daily values, it will be evident to the experienced calculator that a still greater saving of time and labour might be effected if the computations of one year could be made subservient to those of the following years. This may be readily done with respect to the aberration: since the place of the sun on any given day will never differ more than 15 or 16 minutes from its place on the same day in any contiguous year: and by a previous arrangement of the tables, the cor-

* Phil. Mag. Nov. 1826.

New Series. Vol. 1. No. 2. Feb. 1827. 
rection for such difference may be easily applied. The solarnutation likewise, depending on twice the same argument as the aberration, may be conveniently added thereto: and the precession, being a constant quantity, and proportional to the time elapsed, may also be easily united to the aberration. It follows therefore that tables of precession, aberration and solar-nutation for each Greenwich star may be so formed, for every tenth day of the year, as to last for many years to come, without the necessity of any other correction than such as arises from the position of the sun on the given day: and the practical astronomer may at any time take out, almost by inspection, the proper quantities required. With respect to the lunar-nutation, (to which I shall presently refer,) its value will differ from year to year so considerably, that annual computations must be made for each year, and for each star. But, since four computations for each star, in every year, will be sufficient, they may be so arranged from year to year as to be united with the daily values above alluded to. If Mr. Herschel's tables, therefore, be considered as the first step towards the formation of these daily corrections of the stars, I think that Tables, constructed agreeably to the arrangement here alluded to, may be fairly considered as the second step towards that desirable object.

3. The subject of the present communication is to point out the best mode of making this arrangement: and in doing this, I must again advert (as I have so frequently done before) to the valuable labours of M. Bessel, who has, in his Fundamenta Astronomia, page 67, shown the principle upon which this arrangement is made. But, as a more detailed explanation of it may be acceptable to many of your readers, and may probably lead to a more extensive adoption of it in this country, I hope I need not apologize for the space which I must necessarily occupy for this purpose in your interesting Journal.

4. Special tables, for determining the apparent places of the Greenwich stars, are generally so constructed as to show the position of the given star at the moment of its culmination: that being the time at which these stars are more usually observed. And this is the method which I shall adopt in the subsequent investigation*. Now, in order to deduce the values of the corrections from one year to another, a fictitious year must be assumed, commencing at a given epoch; and consisting of $366 \frac{1}{4}$ sidereal days. M. Bessel has assumed the epoch of the sun's mean longitude at mean noon at Paris, on

* In the Nautical Almanac, however, the apparent places are given for noon. 
January 0,1800 , being exactly $280^{\circ}$ : but, in order to preserve uniformity in the arrangement of the tables published by the Astronomical Society, I shall assume the epoch of the sun's mean longitude at mean noon at Greenroich on January 1, 1800 , being exactly $281^{\circ}$ : which, in fact, differs very little from the preceding assumption. The mean motion of the sun in a sidereal day is $58^{\prime} 58^{\prime \prime}, 6417$; which, in 10 such days, amounts to $9^{\circ} 49^{\prime} 46^{\prime \prime}, 417$ : and it will be seen that the intervals of computation may be extended to 10 days, without any risk of error.

5. This being premised, we shall find that the mean longitude of the sun, when any given star culminates, on any given day of the fictitious year, will be equal to

$$
280^{\circ} 13^{\prime} 57^{\prime \prime} ; 88+(d+\alpha) 58^{\prime} 58^{\prime \prime}, 6417
$$

where $\alpha$ denotes the right ascension of the given star (in time), expressed in the fractional part of a day (or $24^{\mathrm{h}}$ ): and $d$ the number of sidereal days, reckoned from the given epoch to the given day*. Consequently the mean longitude of the sun for the moment of culmination of any given star for every 10th day of the fictitious year will be $\dagger$

$$
\begin{aligned}
& 280^{\circ} 13^{\prime} 57^{\prime \prime}, 88+\alpha 53^{\prime} 58^{\prime \prime}, 64+ \\
& \text { for January } 1=0^{\circ} 0^{\prime} \quad 0^{\prime \prime} 0 \\
& 11=94946,4 \\
& 21=193932,8 \\
& 31=292919,2 \\
& \text { February } 10=3919 \quad 5,6 \\
& \text { \&c. \&c. \&c. \&c. }
\end{aligned}
$$

The sun's mean longitude for each star, being found in the manner thus described for every tenth sidereal day, we must apply the equation of the centre, in order to determine the corresponding true longitude of the sun at the same periods; which will be the Argument for finding the aberration. And twice this quantity will be the Argument for finding the solarnutation.

6. But we may readily form a table o the true longitude of the sun corresponding to every degree of his mean longitude in the fictitious year; which will last for many years to

* For those stars, therefore, whose right ascension is between $0^{\mathrm{n}}$ and $18^{\mathrm{h}} 44^{\mathrm{m}}$, the time of culmination, will refer to the preceding day.

+ As an example, take the case of a Aquilce, the mean right ascension of which star, for January 1, 1830, is $19^{\mathrm{h}} 42^{\mathrm{m}} 29^{\mathrm{s}}=-821$ : and the mean longitude of the sun at the time of its culmination on that day is consequently $280^{\circ} 13^{\prime} 58^{\prime \prime}+48^{\prime} 26^{\prime \prime}=281^{\circ} \mathrm{O}^{\prime} 24^{\prime \prime}$. This value being added to $9^{\circ} 49^{\prime} 46^{\prime \prime}$, and its multiples, will give the mean longitude of the sun at the time of its culmination on every subsequent tenth sidereal day of the year. 
come. For, since the place of the sun's perigee in 1830 will be exactly $280^{\circ}$, and since it varies only $62^{\prime \prime}$ from year to year, a table of the equation of the centre computed for the first $90^{\circ}$ of the sun's mean anomaly will answer for the whole circle: attention being paid to the signs. Let $z$ denote the mean anomaly of the sun for the year 1830, then will the equation of the centre be

$$
+1^{\circ} 55^{t} 22^{\prime \prime}, 81 \sin z+72^{\prime \prime}, 61 \sin 2 z+1^{\prime \prime}, 06 \sin 3 z
$$

7. Having thus determined the true longitude of the sun, for each star, on every tenth sidereal day of the fictitious year, we much enter Mr. Herschel's tables with the Arguments

$$
(\odot+N) \text { and }\left(2 \odot+N^{\prime \prime}\right)
$$

and find the respective values of the quantities required. The values of $\mathrm{N}$ and $\mathrm{N}^{\prime \prime}$ are given by Mr. Herschel*.

8. With respect to the precession, or rather the annual variation, its amount at any given moment of time will be expressed by

$$
V \times \frac{\mathbf{L}-28 \mathbf{1}^{\circ}}{360^{\circ}}
$$

where $\mathrm{V}$ denotes the annual variation, and $\mathrm{L}$ the mean longitude of the sun as above determined + . If we substitute the value of $\mathbf{L}$ for each star, and make the proper reductions, this formula will becomef

$$
\begin{aligned}
& \mathrm{V} \times(\cdot 00273 \alpha-00213)+ \\
& \text { for January } 1=00000 \mathrm{~V} \\
& 11=\cdot 00273 \mathrm{~V} \times 10 \\
& 21=\cdot 00273 \mathrm{~V} \times 20 \\
& 31=\cdot 00273 \mathrm{~V} \times 30 \\
& \text { February } 10=\cdot 00273 \mathrm{~V} \times 40 \\
& \text { \&c. \&c. \&c. }
\end{aligned}
$$

$\alpha$ being, as before, the right ascension of the star, converted into the decimal part of $24^{\mathrm{h}}$. These values being added to

* As an example, take the case of \& Aquila on the tabular April 11th. The true longitude of the sun on that day, at the time of the culmination of the star, will be $19^{\circ} 20^{\prime} 6^{\prime \prime}+1^{\circ} 53^{\prime} 27^{\prime \prime}=21^{\circ} 13^{\prime} 33^{\prime \prime}$. Consequently by Mr. Herschel's tables the amount of the aberration in right ascension will be $-0^{s} \cdot 0600$; and of the solar-nutation -0 s. 0536 .

+ Mr. Herschel's Table I. gives the annual variations for mean solar days, and not for sidereal days.

$\ddagger$ As an example, take the case of $\alpha$ Aquile, whose annual variation (in right ascension) is equal to $2: 924$; the proportional part of ${ }^{*}$ which, on January 1 , at the time of its culmination, will be $+28.924(\cdot 00224-$ $\cdot 00213)=+0^{s} \cdot 00032$ : which being added to $0^{s} .0798$, and its multiples, will give the amount of the annual variation at the time of its calmination an crery subsequent tenth sidereal day of the year. Thus, on the tabular April 11 th, it will be $+0^{s} .7985$. 
the respective values of the aberration and solar-nutation for each star, on those days, will give the total amount of the correction, depending on those quantities, for each star at the moment of its culmination on every tenth day of the fictitious year, commencing from that moment of time when the sun's mean longitude at mean noon at Greenwich is assumed as exactly $281^{\circ}$.

9. Having thus deduced the sum of the values of annual variation, aberration and solar-nutation for each star, and for every tenth day of the fictitious year, from January 1 to December $37^{*}$; they must be arranged into tables, with their differences annexed, and are then ready for use. It is in this manner that M. Bessel has computed and arranged his subsidiary tables for the corrections (in right ascension) of 36 Greenwich stars, inserted in the Konigsberg Observations for 1818; and which may be found in Dr. Pearson's valuable Astronomical Tables, pages 149-152. And it is from those subsidiary tables that M. Schumacher annually computes and publishes his apparent places of the Greenwich stars. It is in this manner also that M. Bessel has computed and arranged the Tables in his Fundamenta Astronomice, page 72, for determining the corrections (in right ascension) of 14 prin. cipal Greenwich stars, for the reduction of Bradley's observations.

10. All these tabular values are computed, as I have already observed, for a fictitious year (of $366 \frac{1}{4}$ sidereal days) which commences from an assumed epoch, depending on a given mean longitude of the sun. But, since the mean longitude of the sun at mean noon at Greenwich on January 1, 1800 , is not exactly $281^{\circ}$; and since it is never the same at the commencement of each civil year, a correction is required for reducing the values, in the proposed tables, to the true epoch, according to the civil mode of reckoning time. And a further correction is likewise required for the longitude of the place of observation; on the presumption that the proposed tables may be used on a different meridian from that of Greenwich. These two corrections are precisely similar to those which I have pointed out in the Introduction to the Nero Tables for facilitating the computation of Precession, Aberration and Nutation, alluded to at the commencement of this communication : and therefore it will be unnecessary to dwell further upon them in this place.

* The year is continued to the fictitious date of December 37, in order to complete the decades, and thus facilitate the computation of the differences. For a similar reason the computation of the lunar-nutation is extended to December 67. 
11. There is, however, another correction necessary in the application of these tables, to which I have not yet alluded. It is well known to all practical astronomers that every star will once in every year culminate twice in a mean solar day, when the sun has the same right ascension as the star: and the fictitious day will then have gained a.day on the civil mode of reckoning astronomical time. This correction is common to all the stars: and when the annual values are computed it is usual to annex an asterisk to the interval which includes the day above alluded to; and the intervals, so marked, will comprehend eleven culminations of the star. For those stars also whose right ascension is between $0^{\mathrm{h}}$ and $18^{\mathrm{h}} 44^{\mathrm{m}}$ we must make a fiurther addition of unity to the given date from the commencement of the year to the day on which it is in conjunction with the sun. These corrections M. Bessel denotes by the letter $i$ : so that the Argument for entering the tables will be

$$
\mathbf{T}=\tau+i-x-l,
$$

where $\mathbf{T}$ denotes the given day, according to the civil mode of reckoning astronomical time, from noon to noon: $\tau$ the same nominal date in the tables; $i$ a number which must be taken equal to 0,1 or 2 , according to the circumstances of the case*; and $x$ and $l$ the same as in my Introduction to the New Tables of Precession, Aberration, \&c. already alluded to.

12. These three corrections, - viz. $1^{\circ}$ for the commencement of the year; $2^{\circ}$ for the day of culmination with the sun; and $3^{\circ}$ for the longitude, - are all that are required in the use of these tables. The argument being once found for the given year, the requisite differences for the computation of the annual tables are easily deduced, in most cases by inspection, and always very readily by the assistance of a small auxiliary table of proportional parts.

13. The lunar-nutation may be computed for intervals of 100 days only: for, the motion of the moon's node is so slow that it will be unnecessary to compute for any smaller intervals. The mean longitude of the moon's node on January 1, 1800 , when the mean longitude of the sun was $281^{\circ}$, was, by the recent tables of M.Damoiseau, equal to $33^{\circ} .2107$ : and the

* If the right ascension of the star is greater than $18^{\mathrm{h}} 44^{\mathrm{m}}, i$ is equal to 0 , from January 1 to the day on which the sun's right ascension is the same as that of the star; and, after that period, it is equal to 1 , to the end of the year. If the right ascension of the star is less than $18^{\mathrm{h}} 44^{\mathrm{m}}$, $i$ is equal to 1 from January 1 to the day on which the sun's true right ascension is the same as that of the star; and, after that period, it is equal to 2 , to the end of the year. Thus, for $\alpha$ Aquilce, April 10th, according to the civil mode of reckoning astronomical time, will be equal to the $t a$ bular April 11 th, because in this case $i$ is equal to 1 . 
mean motion of the moon's node in a tropical revolution of the sun being $-19^{\circ} .3417$, we may obtain, by simple addition, the mean place of the moon's node on January 1, of any subsequent fictitious year, commencing when the sun's mean longitude at mean noon is $281^{\circ}$. The mean motion of the nodes, in 100 sidereal days, is $-5^{\circ} \cdot 281$. But these days should (for each star) be computed from the moment of time when the sun's mean longitude is equal to

$$
\begin{aligned}
& 280^{\circ} 13^{\prime} 57^{\prime \prime}, 88+\alpha \times 58^{\prime} 58^{\prime \prime}, 6417 \\
= & \operatorname{Jan}^{\mathrm{y}} 0^{\mathrm{d}} \cdot 21944+\alpha
\end{aligned}
$$

$\alpha$ denoting, as before, the right ascension of the given star expressed in the fractional part of a day. If therefore $\delta 8$ denote the mean place of the moon's node, for any given star, computed for the epoch January $0^{\mathrm{d} .21944}+\alpha$, we shall have the mean places of the moon's node, for the respective periods as under *: viz.

$$
\begin{aligned}
& \text { for Jan. } 1=8 \\
& \text { April } 11=8-5^{0.281} \\
& \text { July } 20=8-10 \cdot 562 \\
& \text { Oct. } 28=8-15 \cdot 843 \\
& \text { Dec. } 67=8-21 \cdot 124
\end{aligned}
$$

The year in every case being supposed to commence when the mean longitude of the sun at mean noon at Greenwich, on January 1,1800 , is presumed to be exactly $281^{\circ}$. But, it will be found that great accuracy in this respect is not essentially necessary, when it concerns only the lunar-nutation.

14. The mean place of the moon's node being computed for those periods in any given year, we may readily deduce the place of the node for the same days in any following year, by merely adding $-19^{\circ} .3417$ to each of such values: this being (as I have already observed) the motion of the moon's node in a tropical revolution of the sun.

15. Having thus determined the mean longitude of the moon's node for every hundredth day of the year, we must enter Mr. Herschel's iables with the Arguments

$$
\left(\Omega+\mathrm{N}^{\prime}\right) \text { and }\left(2 \Omega+\mathrm{N}^{\mathrm{iv}}\right)
$$

and having deduced the lunar-nutation for those days, we may

* As an example, take the case of a Aquila, whose right ascension, reduced to the fractional part of 24 hours, has been already deduced equal to 82118 ; consequently we must compute the mean place of the moon's node for January $0 \cdot 21944+\cdot 82118=$ January $1 \cdot 04062$. The position of the moon's node for that moment of time is $172 \circ .955$; which being added to $-5^{0.281}$, and its multiples, will give the position of the moon's node, at the time of the cumination of the star, on every subsequent hundredth sidereal day of the year. Thus, on the trbular April 11th, it will be $167^{\circ} \cdot 674=167^{\circ} 40^{\prime} 26^{\prime \prime}$. 
readily determine the amount for every tenth day, when we wish to apply it to the computation of the annual tables*. The values of $\mathrm{N}^{\prime}$ and $\mathrm{N}^{\mathrm{i}}$ are given by Mr. Herschel. M. Bessel has, in the formation of his subsidiary tables, added the mean place of the star, at the commencement of the year, to the amount of the lunar-nutation: by which means he saves the computer the trouble of one addition. But, with deference to so great an authority, I would suggest the propriety of keeping those quantities distinct.

16. Having thus given a sketch of the manner in which auxiliary tables may be formed, so as to render Mr. Herschel's tables more generally applicable and useful, and at the same time to enable us to obtain more easily the necessary corrections for the Greenwich stars, I trust that some one will be induced to pursue the subject still further, and endeavour to procure the actual computation of such auxiliary tables for the correction of all the principal stars now observed at the Observatory at Greenwich. The number of those stars was formerly 36 ; and these are the stars whose corrections in right ascension have been tabulated by M. Bessel: the corrections in declination being still a desidexatum. The number in Mr. Herschel's tables is 46: and the whole of these might be tabulated in the manner here proposed, without any considerable trouble. But Mr. Pond has recently extended his list to 60 stars, whose apparent places are now given annually in the Nautical Almanac: and at the end of the volume for 1829 , is given a Catalogue of one hundred principal fixed stars. Whether it is intended to give the apparent places of the whole of these, I know not; but it is evident that the more the list is extended, the more desirable it will be to save the time and labour of the computer: and in no way can this be so effectually done as by the tabular arrangement here proposed.

As your Journal appears to be very extensively circulated on the continent, as well as in this country, I beg leave to take this opportunity of correcting a slight typographical error in my Introduction to the Nero Tables for facilitating the computation of Precession, Aberration and Nutation, which may probably mislead some persons who may employ those tables for a different meridian to that of Greenwich. The error occurs in page $\mathrm{xx}$, where the accent has been placed on the wrong $h$ : therefore in line 10 for $(h)$ read $\left(h^{\prime}\right)$, and in line

* As an example, take the case of $\alpha$ Aquilce on the tabular April 11th. These values will be found equal to $-0^{s} 2065+0^{5} 0039=-0^{s} \cdot 2026$. 
12, for $h^{\prime}$ read $h_{0}$. The subsequent formula, in line 14, will then be accurate: but the cases mentioned in page $\mathrm{xxv}$ require a slight correction, and should be as follow:

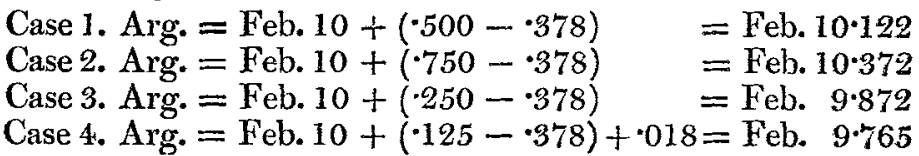

This error has likewise led to the inaccurate expression $x-l$ in pages xxii line 18, xxiii line 9 , xxiv lines 1 and 5 , and xxv line 10; in each of which places it ought to be $x+l$.

It is evident that this error will not affect the argument of the Tables, when they are used in this country, or at any of the observatories in the neighbouring states. But, as it i: ight probably mislead a computer under a more distant meridian, unless previously detected, I have taken the earliest opportunity of making the error known; although it is manifest that the effect will seldom be of much importance.

Jan. 23, 1827.

Francis Bayly.

XXI. Investigation of the Heat extricated from Air when it undergoes a given Condensation. By J. Ivory, Esq. M.A. F.R.S.*

CONCEIVE a quantity of air confined in a close vessel, and let heat be applied to it, the pressure remaining invariable, till it is expanded to a given volume. Again, taking the same mass of air in its first state, let the dimensions of the vessel be suddenly enlarged till the air has acquired the same volume to which it was before expanded by heat: the air within the vessel will become colder, and after a short moment of time will resume its first temperature. We must therefore infer that air, when its volume is increased, absorbs heat, which occasions the coldness; and that the coldness disappears because the loss of temperature is supplied by the communication of heat from the surrounding bodies. That this is a true account of the matter, and that no heat is lost, it is easy to prove; for if the vessel containing the expanded air be reduced to its original bulk, the heat before absorbed will be extricated as the air contracts, producing a rise of temperature which is soon dissipated. Now let heat be applied to the expanded air, while its volume is kept from changing, till the temperature is raised to the same degree as in the first operation: it it evident that the air will now be in

* Communicated by the Author.

Nero Series. Vol. 1. No. 2. Feb. 1827. 